\title{
The Pulsar Birth Rate from the Parkes Multibeam Survey
}

\author{
N. Vranesevic \\ University of Sydney, School of Physics, NSW 2006, Australia, \& \\ ATNF-CSIRO, P.O. Box 76, Epping, NSW 1710, Australia
}

\begin{abstract}
We report on calculations of the pulsar birth rate based on the results of the Parkes multibeam survey. From the observed sample of more than 800 pulsars, we compute the pulsar current, accounting as accurately as possible for all known selection effects. The main goal of this work is to understand the pulsar birth rate as a function of the surface dipole magnetic field strength. We show that pulsars with magnetic fields greater than $10^{12.5} \mathrm{G}$ account for about half of the total birth rate.
\end{abstract}

\section{Introduction}

The Parkes multibeam pulsar survey covers a $10^{\circ}$-wide strip of the southern Galactic plane from $l=260^{\circ}$ to $l=50^{\circ}$ (Manchester et al. 2001). It utilizes a 13 -beam receiver operating in the $20-\mathrm{cm}$ band on the Parkes $64-\mathrm{m}$ radio telescope and is seven times more sensitive than any previous large-scale survey. The survey has discovered more than 600 new pulsars so far, including many that are young and energetic. ${ }^{1}$ In the following analysis we used 520 pulsars that were discovered by the Parkes survey, of which 415 are obtained from the public catalog and 105 are currently unpublished. These new discoveries, together with 210 previously known pulsars, result in a sample of $N_{\mathrm{psr}}=730$ pulsars, with a mean period of $0.76 \mathrm{~s}$.

\section{Pulsar Current Calculation}

The observed distribution of pulsars in the Galaxy differs systematically from the true distribution due to various observational selection effects inherent in pulsar surveys (see the contribution by Lorimer, these proceedings, for further details). We have accurately modeled the sensitivity threshold of the multibeam survey due to these effects and use this model to compute a weight or scale factor for each pulsar by: (1) placing it at large number of randomly selected locations in the model Galaxy; (2) calculating the effective dispersion measure and the interstellar scattering; and (3) recording the number of detections, i.e. those positions for which the predicted flux density exceeds the survey limit. For each pulsar, then, the scale factor is simply the ratio of the total number of

\footnotetext{
${ }^{1}$ Already published pulsars can be found in the on-line catalog at http://www.atnf.csiro.au/research/pulsar/psrcat .
} 
locations in our model Galaxy (typically $10^{5}$ ) to the number of detections. This results in an estimate of the number of similar pulsars in the Galaxy.

The pulsar current, $J$, is the flow of objects from short to long spin periods. We can compute $J$ as a function of period, $P$, by binning pulsars into intervals of width $\Delta P$. The current in a given bin is given by:

$$
J(P)=\frac{1}{\Delta P}\left(\sum_{i=1}^{n_{\mathrm{psr}}} \frac{S_{i} \dot{P}_{i}}{f}\right),
$$

where $S_{i}$ is the scale factor for each pulsar, $n_{\mathrm{psr}}$ is the number of pulsars in the bin and $f$ is the fraction of $4 \pi$ sr covered by the emission beam. In this analysis, we assume for simplicity that $f=0.2$. Under steady-state conditions, the maximum value of $J$ is the birth rate of pulsars. The total number of active pulsars in the Galaxy is given by the sum of $S_{i} / f$. For further details, see Lorimer (these proceedings) and also Vivekanand \& Narayan (1981).

\section{Results}

The results are summarized in Table 1 for both the TC93 distance model (Taylor \& Cordes 1993) and the newer NE2001 model (Cordes \& Lazio 2002). Our main conclusion is that pulsars with surface dipole magnetic fields greater than $10^{12.5} \mathrm{G}$ account for half of the total birth rate. For both distance models, the birth rate of these high-field pulsars is $52 \%$ of the total population. Low-field $\left(<10^{12} \mathrm{G}\right)$ pulsars contribute only $10 \%$ of the total birth rate using the TC93 model, or $17 \%$ for NE2001. Full details of this analysis are now being prepared for publication.

Table 1. Pulsar birth rate and total Galactic population for four sets of data: total plus three ranges of magnetic field strengths.

\begin{tabular}{llccrr}
\hline & $\begin{array}{c}\text { No. in } \\
\text { Sample }\end{array}$ & $\begin{array}{c}\text { Birth Rate } \\
\left(\text { Century }^{-1}\right)\end{array}$ & $\begin{array}{r}\text { Birth } \\
\text { Interval }\end{array}$ & $\begin{array}{r}\text { No. in } \\
\text { Galaxy }\end{array}$ \\
\hline TC93 & total & 723 & $2.53 \pm 0.70$ & $31-55 \mathrm{yr}$ & 259000 \\
& $\log (B) \geq 12.5$ & 166 & $1.32 \pm 0.58$ & $53-135 \mathrm{yr}$ & 11000 \\
& $12.0 \leq \log (B)<12.5$ & 301 & $0.95 \pm 0.34$ & $78-164 \mathrm{yr}$ & 38000 \\
& $\log (B)<12.0$ & 256 & $0.26 \pm 0.16$ & $238-1000 \mathrm{yr}$ & 210000 \\
\hline NE2001 & $\operatorname{total}$ & 721 & $4.50 \pm 1.16$ & $18-30 \mathrm{yr}$ & 660000 \\
& $\log (B) \geq 12.5$ & 168 & $2.35 \pm 0.91$ & $31-69 \mathrm{yr}$ & 17000 \\
& $12.0 \leq \log (B)<12.5$ & 299 & $1.40 \pm 0.49$ & $53-110 \mathrm{yr}$ & 80000 \\
& $\log (B)<12.0$ & 254 & $0.75 \pm 0.35$ & $91-250 \mathrm{yr}$ & 563000 \\
\hline
\end{tabular}

\section{References}

Cordes, J. M., \& Lazio, T. J. W. 2002, astro-ph/0207156

Manchester, R. N. et al. 2001, MNRAS, 328, 17

Taylor, J. H., \& Cordes, J. M. 1993, ApJ, 411, 674

Vivekanand, M., \& Narayan, R. 1981, J. Astrophys. Astr., 2, 315 